\title{
Secondary glaucoma due to thrombosis of sigmoid and transverse sinus
}

\author{
Matta Rudhira Reddy, Vijaya Pai H. \\ Department of Ophthalmology, Katsurba Hospital, Manipal Academy of Higher \\ Education, Manipal, India
}

\begin{abstract}
An 88-year-old female presented with redness in the left eye of one-month duration. On examination, the left eye showed $3 \mathrm{~mm}$ of proptosis with dilated and tortuous episcleral vessels and relative afferent pupillary defect. Intraocular pressure was $60 \mathrm{mmHg}$ and showed open angles on gonioscopy with cup disc ratio of 0.8 in OS. A diagnosis of secondary open-angle glaucoma due to elevated episcleral venous pressure (EVP) was made. Magnetic resonance venogram revealed thrombosis of transverse and sigmoid sinus on the left side. This is the first case report of secondary open-angle glaucoma due to elevated EVP following thrombosis of transverse and sigmoid sinus.
\end{abstract}

Keywords: glaucoma, raised episcleral venous pressure, venous thrombosis

\section{Introduction}

Elevated episcleral venous pressure (EVP) is one of the causes of secondary open-angle glaucoma. Any condition that raises EVP also raises intraocular pressure (IOP) by obstructing the post-trabecular flow of aqueous humor. ${ }^{1}$ The causes of glaucoma secondary to elevated EVP fall into three categories: (a) arteriovenous anomalies, (b) venous obstruction, and (c) idiopathic variety. ${ }^{2}$ Most of the cases are idiopathic, often without angiographic abnormalities. ${ }^{3}$ Cases of glaucoma secondary to elevated EVP due to cavernous sinus thrombosis have been reported previously. ${ }^{4}$ Here, we are reporting a case of secondary open-angle glaucoma, presenting as the initial manifestation of transverse and sigmoid sinus thrombosis without any neurological manifestations.

\section{Case report}

An 88-year-old female presented with chief complaints of redness in the left eye for one month. Redness was not associated with pain, headache, and discharge. There was no history of head injury. She had undergone clear corneal phacoemulsification

Correspondence: Vijaya Pai H., Department of Ophthalmology, Katsurba Hospital, Manipal Academy of Higher Education, Tiger Circle Road, Madhav Nagar, Manipal, Karnataka 576104, India.

E-mail:paivijaya@yahoo.co.in 


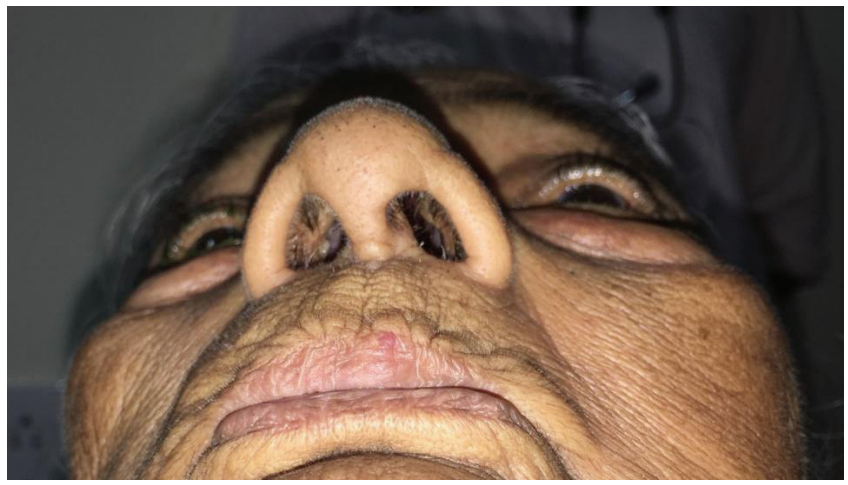

Fig. 1. Proptosis of the left eye.

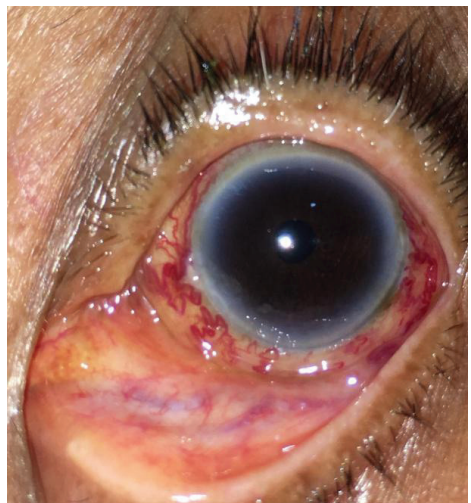

Fig. 2. Dilated and tortuous episcleral vessel OS.

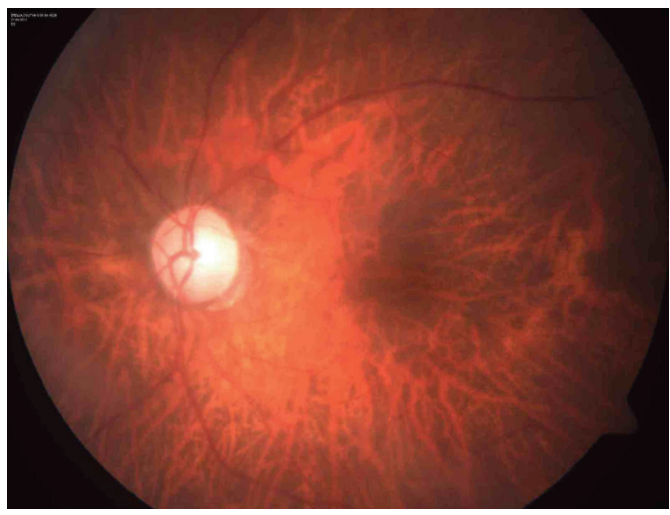

Fig. 3. Optic disc stereo photograph of the left eye showing cup disc ratio of $0.8 \mathrm{OS}$ with disc pallor.

and foldable intraocular lens implantation by the author in the left eye a year ago. Patient was on tablet amlodipine $5 \mathrm{mg}$ for systemic hypertension for the past five years. On examination, her best corrected visual acuity was 6/9 OD and 6/6p OS. The left eye showed proptosis of $3 \mathrm{~mm}$ with Hertel's exophthalmometer (Fig. 1). On auscultation, there was no bruit heard. The left eye showed dilated and tortuous episcleral vessels (Fig. 2), relative afferent pupillary defect, and pseudophakia. Anterior chamber was of normal depth and there was no flare or cells. The right eye examination showed nuclear sclerosis. IOP was $16 \mathrm{mmHg}$ OD and $60 \mathrm{mmHg}$ OS with Goldmann applanation tonometer. Gonioscopy revealed open angles in both eyes, with blood in the Schlemm's canal of the left eye.

Dilated fundus examination showed cup disc ratio of 0.3 OD and 0.8 OS with disc pallor (Fig. 3). 


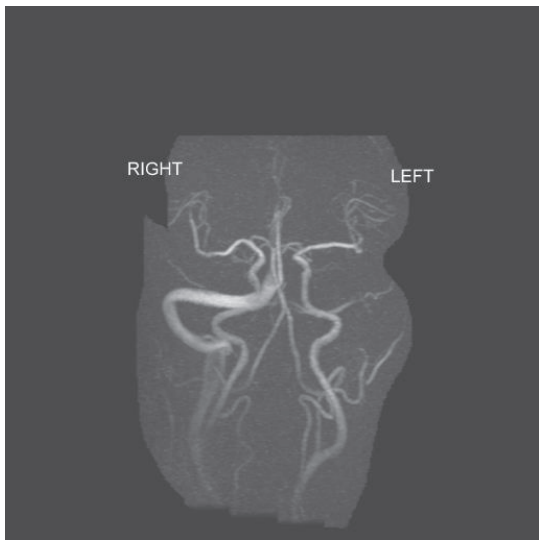

Fig. 4. Magnetic resonance angiogram showing no evidence of AV fistula, nonfilling of transverse sinus on the left side.

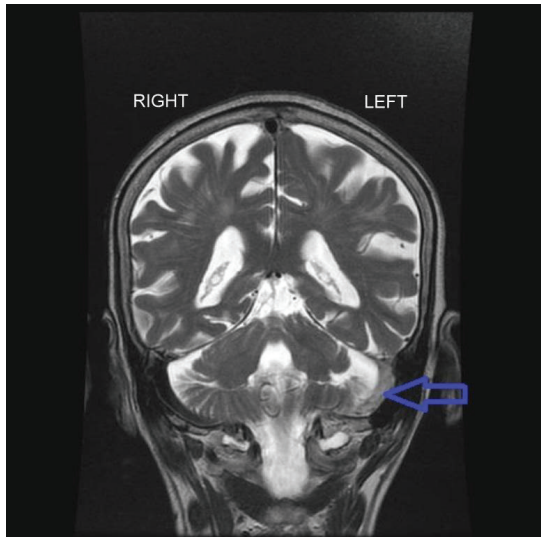

Fig. 5. Magnetic resonance imaging showing thrombus in the left sigmoid and transverse sinus.

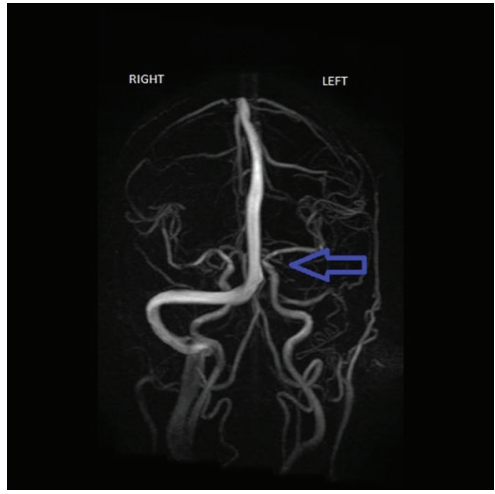

Fig. 6. Magnetic resonance venogram shows no flow in sinuses on left sigmoid and transverse sinus suggestive of thrombosis.

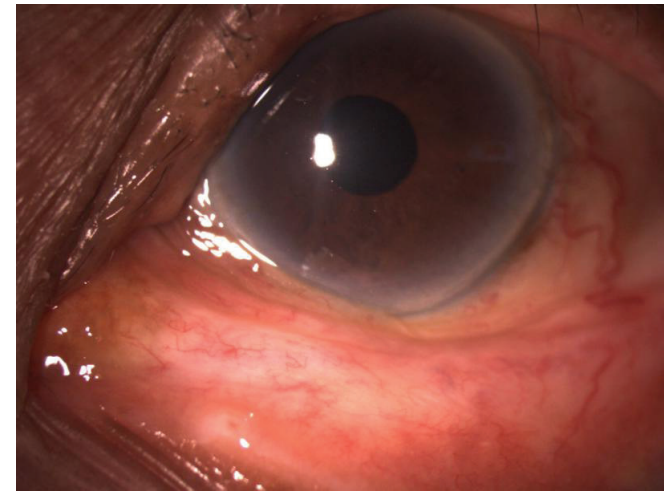

Fig. 7. Posttreatment-decreased congestion of episcleral vessels.

A diagnosis of secondary open-angle glaucoma due to elevated EVP OS was made. We thought it could be due to arteriovenous (AV) fistula of low flow type. Magnetic resonance angiogram did not reveal any evidence of AV fistula (Fig. 4). However, left-side transverse and sigmoid sinuses showed poor filling and thrombus (Fig. 5).

Radiologist suggested magnetic resonance venogram to rule out venous thrombosis. Magnetic resonance venogram revealed thrombosis of transverse sinus and sigmoid sinus on the left side (Fig. 6). 
The patient was started on topical on dorzolamide $2 \%$ and Timolol $0.5 \%$ combination eye drops in the left eye. Patient was referred to the physician for further management of the thrombosis of the sinuses. Patient was investigated for coagulopathies and all were negative. Activated partial thromboplastin time, prothrombin time, was normal and lupus anticoagulant was negative. The physician recommended $\mathrm{T}$. aspirin $150 \mathrm{mg} /$ day.

On follow-up, patient IOP reduced to $26 \mathrm{mmHg}$. Episcleral venous congestion reduced (Fig. 7). At four weeks posttreatment, IOP was $18 \mathrm{mmHg}$.

\section{Discussion}

Cerebral sinus venous thrombosis (CSVT) is a rare type of venous thromboembolism. CSVT represents almost 0.5 to $3 \%$ of all the types of stroke, ${ }^{5}$ affecting predominantly younger people. Clinical manifestations of CSVT are variable and not specific, thus making the definite diagnosis difficult. Common manifestations include headache, altered consciousness, focal neurologic deficits, and seizure. The ocular manifestations include obscuration of vision, papilledema. ${ }^{6}$ Our patient presented with dilation of episcleral vessels with raised IOP with no other neurological symptoms, which is unusual. EVP is a component of the normal IOP, and any rise in EVP is associated with the same amount of increase in IOP and typically has a wide open anterior chamber angle, with blood in the Schlemm's canal. The normal EVP is 8 to $10 \mathrm{~mm} \mathrm{Hg}$. When the EVP rises, a similar rise occurs in the IOP. ${ }^{7} \mathrm{AV}$ fistula is the most common cause of secondary open-angle glaucoma due to elevated EVP. ${ }^{8}$ The episcleral venous system mainly drains into the anterior ciliary and superior ophthalmic veins, finally draining into the cavernous sinus. Thus, any disease process that affects this drainage pathway because of structural, occlusive, compressive, or destructive physiopathology may alter the IOP. ${ }^{9}$ Transverse and sigmoid sinus thrombosis causing increase in EVP is not yet reported. Magnetic resonance imaging and computed tomography are useful to diagnose these conditions. MRV features include nonvisualization of the vessel indicating no flow, flow defect, and presence of collaterals at the site of occlusion. ${ }^{10}$ We believe thrombosis of transverse sinus and sigmoid sinus must have caused back pressure on the cavernous sinus, which in turn caused the increase in EVP and secondary glaucoma. Glaucoma associated with raised EVP is difficult to diagnosis and treat. Our patient responded well to topical dorzolamide Timolol combination. IOP decreased from 60 to $26 \mathrm{mmHg}$ in the left eye. Such a remarkable response to topical antiglaucoma medication, possibility of spontaneous resolution of the cerebral venous thrombosis, should be considered. Our patient presented with glaucoma as the initial presentation of cerebral venous thrombosis. We conclude that thrombosis of sigmoid and transverse sinuses is a rare cause of secondary glaucoma. 


\section{References}

1. Stamper RL, Lieberman MF, Drake MV (Eds). Becker-Shaffer's Diagnosis and Therapy of the Glaucomas. 8th ed. St. Louis: Mosby; 2009:284.

2. Weinberg RN, Jeng S, Goldstick BJ. Glaucoma secondary to elevated episcleral venous pressure. In: Ritch R, Shields MB, Krupin T, eds. The Glaucomas. St. Louis: Mosby; 1989:1130.

3. Cioffi GA. Glaucoma: Basic and Clinical Science Course. San Francisco, CA: American Academy of Ophthalmology; 2014-2015:95.

4. De Keizer R. Carotid-cavernous and orbital arteriovenous fistulas: ocular features, diagnostic and hemodynamic considerations in relation to visual impairment and morbidity. Orbit. 2003 Jun;22(2):121-142.

5. Bousser MG, Ferro JM. Cerebral venous thrombosis: an update. Lancet Neurol. 2007;6:162-170.

6. Alvis-Miranda HR, Castellar-Leones SM, Alcala-Cerra G, Moscote-Salazar LR. Cerebral sinus venous thrombosis. J Neurosci Rural Pract. 2013 Oct-Dec;4(4):427-438.

7. Moses RA, Grodzki WJ. Mechanism of glaucoma secondary to increased venous pressure. Arch Ophthalmol. 1985;103:1653-1658.

8. Carvalho RA, Delloiagono HS, Jammal AA, Resende GM, Angotti HS. Secondary glaucoma following carotid cavernous fistula. Rev. Bras. Oftalmol. 2016;75(2):156-159.

9. Nassr MA, Morris CL, Netland PA, Karcioglu ZA. Intraocular pressure change in orbital disease. Sur Ophthalmol. 2009;54(5):519-544.

10. El Damarawy EA, El-Nekiedy AE, Fathi AM, Eissa ED. Role of magnetic resonance venography in evaluation of cerebral veins and sinuses occlusion. Alex J Med. 2012;48:29-34. 